\title{
Le défi de l'optimisation des pratiques professionnelles en santé
}

L'article de Johanne Goudreau et coll. ${ }^{1}$ se place au centre d'une problématique à la fois très actuelle et essentielle de nos systèmes de soins : il s'agit de déterminer les moyens les plus efficients pour que les médecins s'engagent dans une dynamique d'amélioration de leurs pratiques.

Cette dynamique d'amélioration constitue la réponse que les médecins (et plus largement l'ensemble des professionnels de santé) ont adopté pour s'accommoder au mieux des contraintes qui s'appliquent à chaque système de santé, qu'il soit de "culture" publique ou privée. Ces contraintes sont bien connues: elles tiennent à la complexité croissante de l'exercice des professions de santé, lesquelles sappuient sur une matière scientifique et des technologies de plus en plus sophistiquées, au point que les coopérations interprofessionnelles deviennent souvent indispensables. Elles tiennent également aux attentes et aux exigences des patients et des associations qui les représentent. Elles tiennent enfin aux coûts et donc aux financements correspondants, invariablement en augmentation, mais dont la croissance doit être maîtrisée.

Diverses approches et méthodes ont donc été expérimentées depuis le début des années 90 pour mettre en ceuvre le mécanisme vertueux "évaluation/formation $\Rightarrow$ amélioration des pratiques". Cette mise en cuvre était d'autant plus hypothétique que ces expérimentations faisaient souvent l'impasse sur les incitations qui auraient été utiles et laissaient quelquefois persister l'incertitude sur les sanctions pouvant toujours résulter d'une procédure d'évaluation.

Quoi quil en soit, garantir la qualité des soins et améliorer les pratiques médicales constituent désormais deux orientations prioritaires des systèmes de soins de l'ensemble des pays de l'Organisation de coopération et de développement économiques $(O C D E)^{2}$. Ces orientations ont été régulièrement confirmées au cours des quinze dernières années, même si de nombreux praticiens restent dubitatifs devant les incantations répétées et les efforts déployés. En effet, pour nombre de ces médecins, la qualité est déjà une exigence permanente, personnelle, éthique et déontologique, à laquelle ils s'efforcent de satisfaire depuis le jour où ils ont commencé d'exercer.
Pourtant, le mouvement est en route.

Ainsi, une des premières tâches a été d'établir dans tous les domaines cliniques possibles des "états de l'art". Ces états permettent de répertorier et d'analyser l'ensemble des connaissances "prouvées" en médecine; puis les données correspondantes sont synthétisées et regroupées sous forme de recommandations de pratique. Ces recommandations sont alors destinées aux médecins. De leur côté, les anglophones ont initié ces dernières années des démarches comparables, qui se sont traduites par l'élaboration d'innombrables guidelines.

Pour transmettre ces recommandations de pratique ou guidelines aux médecins, les organisations professionnelles et les différents moyens de formation médicale continue ont été mobilisés de manière croissante tout au long des années 90. Las, à la fin de cette décennie d'expérimentation, il semble bien quill soit très difficile de faire évoluer, au terme d'un processus d'information ou de formation permanente, le comportement professionnel des médecins.

Ces difficultés avaient été clairement identifiées dès 1989, quand au moins deux auteurs avaient distinctement signalé le faible rendement des recommandations. C'est d'abord le canadien Jonathan Lomas ${ }^{3}$ qui révélait que les conclusions d'une conférence de consensus ne modifiaient pratiquement pas les taux de césariennes pratiquées par des médecins (alors que ces mêmes médecins indiquaient que leur manière de faire allait être sérieusement modifiée par le consensus). C'est ensuite, l'américain Jeffrey Stross ${ }^{4}$ qui constatait qu'au sein d'un groupe de médecins dont $75 \%$ étaient avertis et tout à fait favorables à une nouvelle stratégie thérapeutique utile, moins de $15 \%$ d'entre eux l'adoptaient rapidement. Depuis lors, ces résultats médiocres ont été presque constamment observés, d'autant plus que les études réalisées ${ }^{5}$ mesuraient non seulement les modifications du comportement des médecins, mais aussi les évolutions de l'état de santé des malades.

Les différentes priorités pour parvenir à une amélioration de la qualité des soins ont donc été réorganisées; si établir (ou actualiser) les états de l'art reste important, toute l'attention est maintenant fixée sur les stratégies quill faut adopter pour que les médecins utilisent les recommandations de pratique. C'est précisément 
dans ce cadre que se situe le travail de Johanne Goudreau et coll. en proposant un programme (la "trousse " qui en France aurait vraisemblablement été appellé "le kit "...), soigneusement élaboré au terme d'une analyse approfondie et faisant appel à un ensemble diversifié de moyens pédagogiques.

Cependant, les comportements des médecins, notamment quand il s'agit de faire des choix, sont déterminés - au-delà du seul savoir-par de nombreuses circonstances et influences résumées ci-dessous sur un schéma établi lors d'un séminaire tenu dès $1995^{\circ}$. C'est pourquoi plusieurs travaux publiés récemment sur les caractéristiques des interventions qui facilitent les modifications de comportement des médecins sont à retenir.

Ainsi, ces travaux ${ }^{7-10}$ font ressortir les points importants suivants :

- Pour un médecin, c'est beaucoup plus en prenant une part active à un processus complet qu'en étant simplement destinataire d'un message pédagogique que la modification du comportement peut survenir. Ce premier point est lourd de conséquence; en fait, cela implique que c'est davantage dans un projet d'exercice professionnel que dans un projet pédagogique que les médecins doivent être appelés à s'engager.

- Tous les problèmes pratiques et contraintes liés à l'exercice médical "sur le terrain" doivent être pris en compte avant d'établir des recommandations et de les soumettre à des praticiens.

- C'est en multipliant les formes et en diversifiant les circonstances sous lesquelles un message parvient au médecin que les chances de l'intégrer dans sa pratique s'accroissent. Ainsi un même message peut être "porté " dans la presse professionnelle, dans la presse grand public, par des leaders locaux, au cours d'ateliers interactifs, par les patients, par les autorités sanitaires, etc.

- Les entretiens individuels au cours desquels les caractéristiques et les motivations personnelles de chaque médecin sont prises en compte, restent très utiles et efficaces. C'est la raison du succès d'approches de type academic detailing ${ }^{I I}$ et c'est sûrement un des motifs pour lesquels l'industrie pharmaceutique attache une telle importance aux visites médicales.
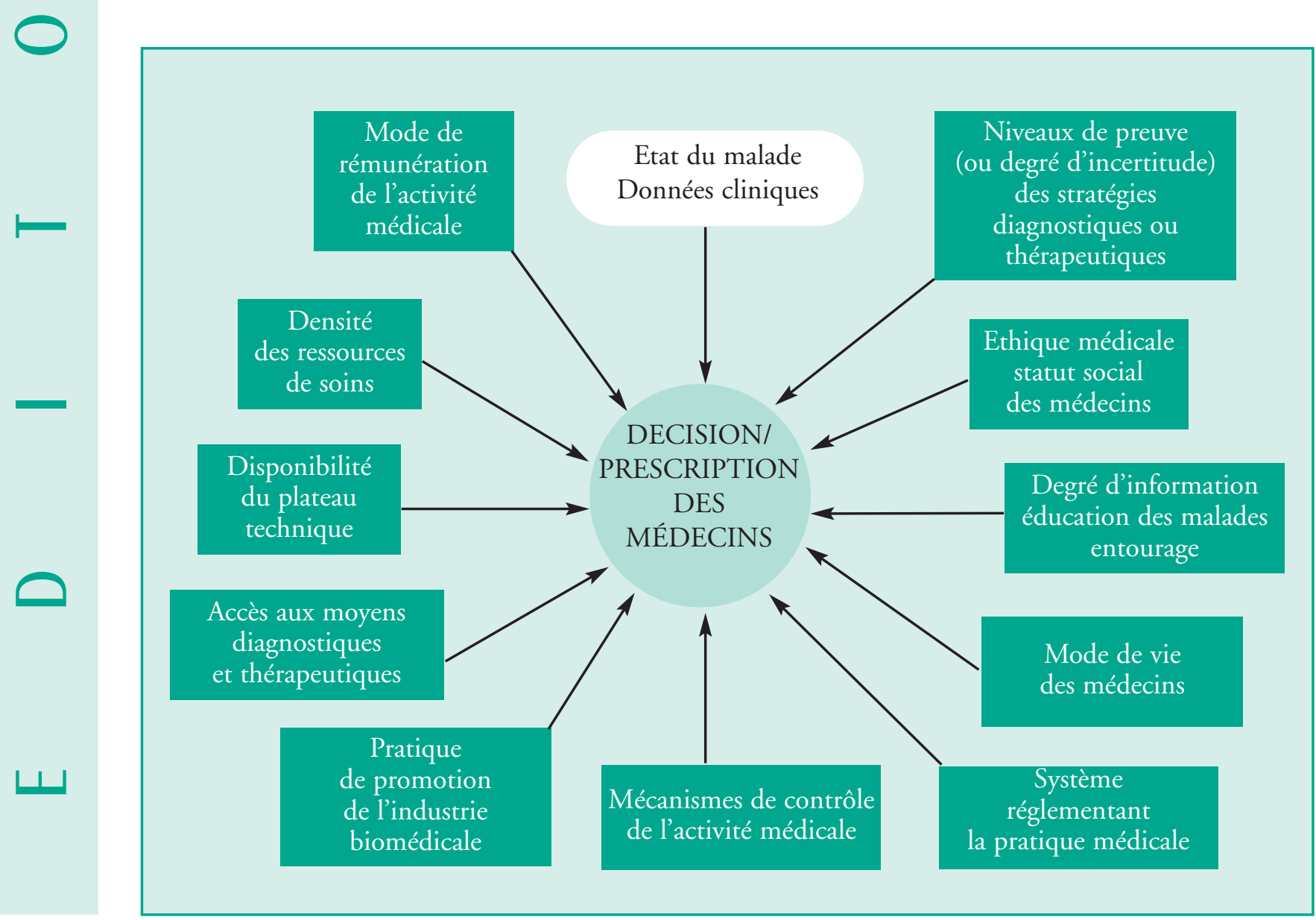
Pour tous les points ci-dessus, des études rigoureuses ont été menées afin de démontrer leur efficacité. Il ne faut cependant pas perdre de vue que cette efficacité n'est qu'une valeur moyenne calculée à partir d'un grand nombre de mesures individuelles. Et bien entendu, chaque mesure individuelle peut s'écarter - très sensiblement - de la moyenne; ce qui signifie que chaque médecin doit rester maître des moyens de sa formation permanente.

Plusieurs auteurs et éditeurs anglo-américains l'ont bien compris. Ils tiennent à la disposition des médecins soit sous forme de documents imprimés* ${ }^{*}$ soit sous forme d'aide mémoires électroniques des synthèses des "états de l'art". Il revient alors à chaque médecin d'y trouver les données pour améliorer sa pratique.

Jean-Michel CHABOT

Haute Autorité de santé, Chef du service Évaluation des Pratiques

2 avenue du Stade de France - 93218 Saint-Denis La Plaine cedex mailto:m.chabot@has-sante.fr

Laboratoire de santé publique

de la faculté de médecine de Marseille Université de la Méditérrannée (Aix-Marseille II)

* Un tel ouvrage intitulé Clinical Evidence (Décider pour traiter), édité par le BMJ Publishing Group en langue anglaise et par les éditions RanD en français, connaît actuellement une diffusion importante dans plusieurs pays.

\section{Références}

1. Goudreau J, Rodrigues I, Lalonde L, Fournier M. Faire connaître les recommandations d'un guide de pratiques : développement et évaluation d'une intervention de formation continue. Pédagogie Médicale 2004; 6 : 147-159

2. Panorama de la santé. Les indicateurs de l'OCDE 2003. [On-line]. Disponible sur : http://lysander.sourceocde.org $/ v l=3246527 / \mathrm{cl}=108 / \mathrm{nw}=1 / \mathrm{rps}$ $v /-6731 / v 2003 n 22 / s 1 / p 1 l$

3. Lomas J, Anderson GM, Pierre KD, Vayba E, Enkin M, Hannah W. Do practice Guidelines guide practice? The effect of a consensus statement on the practice of physicians. N Engl J Med 1989; 321 : 1306-1311.

4. Stross J.K. Relationships between knowledge and experience in the use of disease-modifying antirheumatic agents. A study of primary care practitioners. JAMA 1989 ; 262 : 2721-2723.

5. Worrall $G$, Chaulk P, Freake D. The effects of clinical practice guidelines on patient outcomes in primary care : a systematic review. CMAJ. 1997 ; $156: 1705-12$.
6. ENSP, MIRE. Les transformations des systèmes de santé en Europe : vers de nouveaux contrats entre prestataires, payeurs et pouvoirs publics? Rennes : ENSP, 1995.

7. Davis DA, Thomson MA, Oxman AD, Haynes RB. Changing physician performance. A systematic review of the effect of continuing medical education 1strategies. JAMA 1995 ; 274 : 700-705.

8. Grol R, Dalhuijsen J, Thomas S, Veld C, Rutten $G$, Mokkink H. Attributes of clinical guidelines that influence use of guidelines in general observational study. BMJ 1998 ; 317 : 858-861.

9. Griffiths $C$, Feder $G$. Clinician education : a key to implementing asthma guidelines? QualHealth Care $1999 ; 8: 73-74$.

10. Stross JK. Guidelines have their limits. Ann Intern Med 1999 ; 131 : 304-306.

11. Chabot JM. La Visite Académique. Rev Prat 2001 ; $51: 1687-1688$ 


\section{Quelques questions posées par l'épreuve d'évaluation de la lecture critique d'articles scientifiques}

A l'issue du deuxième cycle de leurs études (études pré-graduées du cursus nord-américain), tous les étudiants en médecine français doivent désormais passer des épreuves classantes nationales (ECN) anonymes ${ }^{1,2}$. Conformément à une conception républicaine bien établie du mérite et de l'équité, le classement à ces épreuves conditionne les choix de chaque étudiant; il détermine à la fois la filière de formation spécialisée à laquelle il pourra accéder ainsi que l'université et le centre hospitalier universitaire de rattachement, au sein desquels il recevra sa formation. Fort logiquement, au cours du deuxième cycle, les étudiants orientent leurs activités vers la préparation des ECN. Une partie importante de cette préparation s'effectue en dehors des facultés, au sein d'officines privées, au détriment toléré et parfois encouragé des enseignements réguliers et des stages hospitaliers; en dépit de ce biais, des palmarès officieux des facultés de médecine françaises circulent, extrapolés imprudemment mais avec complaisance à partir du classement des étudiants. Nonobstant ces évolutions préoccupantes, dont il faudra bien un jour évaluer l'impact sur la qualité des apprentissages des étudiants diplômés et donc sur les compétences des médecins formés, limportant budget que les pouvoirs publics consacrent chaque année à l'organisation des ECN (1,35 millions d'euros) ${ }^{3}$ souligne l'apparent consensus social établi autour de cette modalité d'orientation professionnelle finale des étudiants en médecine en France.

Ces contraintes académiques et sociales étant jusqu’à nouvel ordre acceptées -et leurs conséquences pédagogiques étant implicitement assumées-, il convient au moins que les modalités de l'épreuve satisfassent effectivement les standards de rigueur, garants du principe d'équité qui en fonde la légitimité. L'exigence d'avoir à classer avec fiabilité 4500 étudiants -et probablement 7000 dès $2011^{4}$ ne laisse guère le choix que de convoquer le regard docimologique pour en apprécier les critères. Un tel examen devrait donc être à la fois valide, fidèle et réalisable avec des contraintes acceptables. Consistant actuellement en l'analyse de neuf dossiers cliniques, à propos desquels les étudiants sont interrogés à l'aide de quatre à dix questions à réponse ouverte, il devra comporter en outre, à partir de 2008, une épreuve de lecture critique d'un ou plusieurs articles scientifiques (LCA).

En raison de sa nouveauté, cette dernière épreuve suscite un questionnement qui rend compte d'inquiétudes, tant de la part de certains enseignants que de la plupart des étudiants. Dès 2001, dans ces mêmes colonnes", JeanPaul Fillastre et Raymond Colin formulaient des réserves quant à la décision d'inclure prématurément une telle épreuve, "alors que nous ne disposons pas encore de méthodes valides et fidèles pour évaluer cette capacité" "; ils mettaient en garde contre les "risques importants qui pourraient altérer l'équité de l'examen national et aboutir à des situations de contentieux». En 2002, à la suite d'un mouvement de grève national, les étudiants en médecine obtenaient des ministères de tutelle le report de l'épreuve à 2008, compte tenu d'une préparation à l'exercice quills jugeaient insuffisante et surtout très inégale d'une faculté à l'autre. Depuis lors, les différentes facultés se sont tant bien que mal attelées à la tâche; en se fondant sur des approches pédagogiques issues de leurs cultures institutionnelles propres, elles ont mis en ceuvre des activités d'enseignement, d'apprentissage et d'évaluation de la $L C A$ selon des modalités variables. Seule une minorité de ces expériences fait l'objet d'une évaluation systématique et, à notre connaissance, aucune initiative coordonnée n'a été mise en place pour en mutualiser les résultats et en tirer des conclusions. Il faut donc saluer les initiatives pionnières en ce domaine et remercier les auteurs qui inscrivent ces démarches exploratoires dans une logique scientifique d'évaluation par leurs pairs, en publiant leurs travaux.

L'article de Christine Louis-Sylvestre et coll. ${ }^{5}$, qui paraît dans ce numéro, fait suite au travail de Francis Roussel et coll. ${ }^{6}$, publié dans le précédent. Les résultats des deux études accréditent lidée que, dans l'état actuel de la conception de l'épreuve et de préparation des enseignants chargés de la corriger, l'organisation d'une épreuve de $L C A$ dans le cadre des ECN soulève des questions importantes. Certes, les deux études comportent des limites méthodologiques, que leurs auteurs discutent d'ailleurs fort loyalement. Des spécialistes en orthodoxie docimologique pourraient soubaiter qu'elles aient eu recours à des raffinements expérimentaux et statistiques plus exigeants. Il demeure que des enseignants engagés, soucieux de leur responsabilité sociale, ont montré la voie en sastreignant à une démarche réflexive quant à leurs pratiques d'enseignants et d'évaluateurs. De ce fait, même si la solidité de leur démonstration et la généralisabilité de leurs conclusions ne peuvent être tenues comme définitivement établies, leurs résultats sont suffisamment significatifs pour introduire un doute sérieux quant au critère de fidélité exigible de la correction de l'épreuve de LCA, même quand on utilise une grille de correction. Lapparente sécurité apportée par l'exigence d'une double correction des épreuves ne peut pas complètement rassurer. En stipulant que "chaque dossier, corrigé en double correction indépendante, est noté de 0 à 100 [...] [et que] la note 
retenue est la moyenne arithmétique des deux notes "2, les dispositions réglementaires actuelles acceptent de fait l'infidélité de l'instrument mais formulent une solution très hasardeuse à cet inconvénient, en postulant que la moyenne arithmétique de deux mesures erronées fournira une mesure exacte. En prévoyant que "lorsque, pour un même dossier, l'écart de notation constaté entre deux correcteurs est égal ou supérieur à 15 points, une troisième correction est assurée [et que] dans ce cas, la notation qui en résulte est retenue " ${ }^{2}$, ces dispositions acceptent également le risque qu'une copie victime de deux erreurs de mesure se produisant dans le même sens et avec le même ordre de grandeur ne soit pas révisée. Il n'est pas possible de statuer quant à la validité de l'épreuve puisque son évaluation n'était l'objet d'aucune des deux études rapportées. Il est très positif que les objectifs pédagogiques formulés par le conseil scientifique du centre national des concours d'internat ${ }^{7}$ prennent en compte plusieurs éléments du cadre de référence établi par Nicole Audet et Hélène Leclère, à l'issue d'une recension systématique des écrits, pour rendre compte des habiletés requises pour la LCA en médecine. ${ }^{8}$ On peut cependant émettre l'hypothèse qu'il existe de sérieuses incertitudes quant à la validité de construit des épreuves qui seront proposées. Ce type de validité concerne la justesse et la pertinence avec lesquelles l'épreuve renseigne sur les apprentissages relatifs aux différentes composantes de la " construction" théorique des compétences visées. L'une des difficultés principales tient au fait que les aptitudes cognitives requises sont fortement contextualisées à des situations et à des contenus bio cliniques spécifiques. Des compétences transversales et transférables ne peuvent être construites, grâce à des interventions pédagogiques appropriées, qu'après une exposition itérative à une variété de situations particulières'. Dès lors, l'évaluation de telles compétences, comme le souligne Geoff Norman en conclusion d'une récente revue systématique de la littérature ${ }^{\text {, }}$ soulève de nombreuses questions non résolues qui devraient être abordées dans le contexte d'une démarche de recherche.

L'évaluation des apprentissages est d'abord un acte pédagogique. A cet égard, les enseignants doivent avoir conscience que les éléments quils prennent en compte dans le cadre de leurs évaluations adressent aux étudiants des signaux extrêmement puissants quant aux apprentissages à privilégier. Audelà du contexte français évoqué, les contributions préliminaires de Francis Roussel et Christine Louis-Sylvestre constituent en tout état de cause une invitation expresse à ce qu'un groupe de travail ad hoc sintéresse aux expériences de formation à la LCA et d'évaluation des apprentissages concernés. Dans un esprit d'expérimentation, acceptant que les procédures soient imparfaites mais cherchant constamment à les optimiser, ce groupe pourrait mutualiser les pratiques innovantes, les travaux de recherche évaluative et, à terme, formuler des recommandations. Dans son rôle de société savante, riche des ressources francophones qu'elle peut mobiliser, la Société internationale francophone d'éducation médicale
(SIFEM) pourrait, sur sollicitation, apporter une expertise scientifique et contribuer à valoriser ces travaux.

L'évaluation des apprentissages est aussi une pratique sociale, qui permet à l'institution universitaire d'attester le niveau de connaissances et de compétences développées par les étudiants et de rendre publiques les décisions les concernant. Dans le contexte spécifique des exigences et des contraintes des ECN françaises, il appartient aux responsables universitaires et politiques d'apprécier les réponses à apporter à des difficultés et à des questions désormais plus clairement identifiées.

$$
\begin{array}{r}
\text { Jean Jouquan } \\
\text { mailto:jean.jouquan@chu-brest.fr }
\end{array}
$$

\section{Références}

1. Décret $n^{\circ}$ 2004-67 du 16 janvier 2004 relatif à l'organisation du troisième cycle des études médicales. Journal officiel de la République française 18 janvier 2004: 1394-1395.

2. Arrêté du 29 janvier 2004 relatif à l'organisation des épreuves classantes nationales anonymes donnant accès au 3 cycle spécialisé des études médicales. Journal officiel de la République française du 5 février 2004 : 2519-2521.

3. Projet de loi de finances pour 2005: enseignement supérieur. Rapport général fait au nom de la commission des Finances, du contrôle budgétaire et des comptes économiques de la Nation sur le projet de loi de finances pour2005[Online].Disponiblesur:http://www.senat.fr/ rap/l04-074-325/l04-074-3251.pdf

4. Commission Démographie médicale. Rapport présenté par Berland Y. 2005 [On-line].Disponible sur: http://www.sante.gouv.frl

5. Louis-Sylvestre C, Furham C, Housset B. Difficultés de correction d'une épreuve d'analyse critique d'article scientifique : une étude exploratoire. Pédagogie Médicale 2005;6:xx-yy.

6. Roussel F, Czernichow P, Lavoinne A, Lemeland J-F, Fillastre J-P. Reproductibilité d'une épreuve de lecture critique d'article : évaluation par une étude pilote chez 59 étudiants en médecine. Pédagogie Médicale 2005;6 .71-78.

7. Conseil scientifique $d u$ centre national $d u$ concours d'internat. Epreuve écrite de lecture d'article. 2003[Online]. Disponible sur: http://www. cnci.univparis5. fr/medecine/CritiqueArticle.doc

8. Audet $N$, Leclère $H$. Les habiletés requises pour la lecture critique en médecine: un cadre de référence issu d'une recension des écrits. Pédagogie Médicale 2001;2 :206-212

9. Norman G. Critical thinking and critical appraisal. In: GR Norman, CPM Van der Vleuten, DI Newble (eds). International Handbook of Research in Medical Education. Dordrecht: Kluwer Academic Publishers, 2002:277-298 